\title{
Duloxetine-induced hypomania: case report and brief review of the literature on serotonin-noradrenaline reuptake inhibitors (SNRIs)-induced mood switching
} Vaios Peritogiannis*1,2, Thomas Hyphantis ${ }^{1,2}$, Katerina Antoniou ${ }^{3}$, Vassiliki Mouka $^{1,2}$ and Venetsanos Mavreas ${ }^{1,2}$

\author{
Address: ${ }^{1}$ Mobile Psychiatric Unit of the Prefectures of Ioannina and Thesprotia, Greece, ${ }^{2}$ Department of Psychiatry, Medical School, University of \\ Ioannina, Ioannina, Greece and ${ }^{3}$ Department of Pharmacology, Medical School, University of Ioannina, Ioannina, Greece \\ * Corresponding author
}

from International Society on Brain and Behaviour: 3rd International Congress on Brain and Behaviour

Thessaloniki, Greece. 28 November - 2 December 2007

Published: 17 April 2008

Annals of General Psychiatry 2008, 7(Suppl I):S23I doi:I0.1 I86/I744-859X-7-SI-S23 I

This abstract is available from: http://www.annals-general-psychiatry.com/content/7/SI/S23I

(c) 2008 Peritogiannis et al.; licensee BioMed Central Ltd.

\section{Background}

Manic switching during antidepressant treatment has been reported with every class of antidepressant drugs. SNRIs, namely venlafaxine, milnacipran and duloxetine have been increasingly used for the treatment of unipolar and bipolar depression and are well tolerated and sufficiently effective due to their dual mechanism of action.

\section{Materials and methods}

A case of duloxetine-induced hypomania in a non-bipolar patient is presented, and a brief review of all the cases of SNRIs' induced mania and hypomania has been carried out.

\section{Results}

A 61-year old female patient was examined as an outpatient, presented with a depressive episode which appeared during the previous month, despite continuous, prophylactic treatment with sertraline $200 \mathrm{mg} /$ day. According to her medical records, the patient had a 23-year history of recurrent depression with severe episodes (ICD-10:F33). She also had a hyperthymic/cyclothymic temperament and she occasionally presented with mild paranoid symptoms, unrelated to the episodes of depression. Her family history revealed that two patient's sisters had been institutionalized for unknown severe mental illnesses. Sertraline was tapered and the patient was switched to duloxetine at $60 \mathrm{mg} /$ day. Three days after initiation of duloxetine, the patient became irritable, hypertalkative and aggressive towards her husband. Duloxetine was immediately discontinued and the symptoms started to resolve, but remitted completely only after the introduction of quetiapine. A review of the literature revealed only two reports of duloxetine-induced mania and a total of 13 reports of SNRIs-induced mania/hypomania.

\section{Conclusions}

The available data suggest that SNRIs, especially venlafaxine, can induce mood switching in patients with bipolar depression and in certain patients with unipolar depression, but the potential of duloxetine and milnacipran to induce manic/hypomanic symptoms can not be disregarded. Switching appears to be dose-related and treatment initiation with lower doses and upward titration when needed may be preferable in selected cases and may help minimizing the risk of mood switching.

\section{References}

I. Lim CJ, Leckman JF, Young C, Martin A: Antidepressant-induced manic conversion: a developmentally informed synthesis of the literature. Int Rev Neurobiol 2005, 65:25-52.

2. Desarkar P, Bakhla A, Sihna VK: Duloxetine-induced ultrarapid cycling in an adolescent with bipolar depression. J Clin Psychopharmacol 2007, 27:115-116.

3. Dios C, Ezquiaga E: Manic switching in patients receiving duloxetine. Am J Psychiatry 2007, I 64: I I 2 I.

4. Stoner S, Williams R, Worrel J, Ramlatchman L: Possible venlafaxine-induced mania. J Clin Psychopharmacol 1999, I 9:184-I85. 